\title{
The effect of melatonin supplementation on the quality of sleep and weight status in postmenopausal women
}

\author{
Ewa Walecka-Kapica ${ }^{1}$, Grażyna Klupińska ${ }^{1}$, Jan Chojnacki ${ }^{1}$, Karolina Tomaszewska-Warda², \\ Aleksandra Błońskaํ, Cezary Chojnacki ${ }^{1}$
}

${ }^{1}$ Department of Clinical Nutrition and Gastroenterological Diagnostic, Medical University of Lodz, Poland

${ }^{2}$ Department of Gastroenterology, Medical University of Lodz, Poland

\begin{abstract}
Aim of the study: We evaluated the effect of melatonin supplementation on the nutritional status of postmenopausal women.

Material and methods: The study included 56 women (51-65 years) and 25 healthy women (27-36 years). The emotional state was assessed using Hamilton Depression Rating Scale (HAM-D), the quality of sleep using Insomnia Severity Index (ISI). Body mass index (BMI) and waist-hip ratio (WHR) were also calculated. The patients were divided into 3 groups: group I (control) - 25 women with normal body weight, group II 26 postmenopausal women with normal body weight, group III - 30 postmenopausal women with high body weight. In women from group II and III, routine laboratory tests, levels of thyroid-stimulating hormone (TSH), $17 \beta$-estradiol, prolactin, follicle-stimulating hormone (FSH) and the concentration of 6 -hydroxymelatonin sulphate (6-HMS) in day/night urine fractions were determined. On the day of the examination, women remained on a liquid diet $(1800 \mathrm{kcal})$. Next, a balanced diet of $1500 \mathrm{kcal}$ and $5 \mathrm{mg}$ of melatonin administration were recommended. The follow-up examinations were performed after 4, 8, 12, 16, 20 and 24 weeks.

Results: The patients from groups II and III showed similar mild levels of anxiety and depression and a significant degree of sleep disorders. In group III, lower urinary 6-HMS excretion was observed at night. In both groups a negative correlation was found between urinary 6-HMS excretion and the degree of sleep disorders. After 24 weeks, a statistically significant improvement of quality of sleep was obtained. A negative correlation was detected between urinary 6-HMS excretion and BMI.

Conclusion: Melatonin supplementation contributed to body weight reduction.

Key words: melatonin, menopause, body mass index, sleep disorders.
\end{abstract}

\section{Introduction}

Melatonin (MEL) is a molecule with diverse and still not completely recognized properties, but it mainly demonstrates antioxidant, anti-inflammatory and immunomodulatory activity [1]. Changes in the volume or rhythm of its secretion were observed in many pathological conditions, but it is not always clear whether they are their cause or effect. These doubts concern among others persons with eating disorders.

In one of the first studies on this issue it was found out that the average daily secretion of melatonin is significantly higher in women with both anorexia nervosa and obesity than in the control group [2].

At the same time in these groups, an inverse correlation between the level of melatonin and luteinizing hormone $(\mathrm{LH})$ was observed. It was concluded that the increased secretion of melatonin during daytime could inhibit the gonadal function in these patients and play an important role in the pathogenesis of eating disorders [3].

These preliminary clinical observations were an inspiration for conducting experimental studies on animals, in which, among others, melatonin was found to inhibit insulin secretion [4]. Decreased insulin secretion, increased insulin resistance, development of glucose intolerance and increased body weight were also observed in pinealectomized rats [5].

It was also shown that melatonin supplementation had a beneficial effect on the secretion of leptin, adiponectin and on the level of glucose, cholesterol (LDL) and triglycerides, particularly in animals with induced obesity [6-9]. These changes occur simultaneously with the reduction of both appetite and body weight [10-13]. These beneficial effects were obtained using a dose of $4 \mathrm{mg} / \mathrm{kg}$ of melatonin for 16 weeks [9], $10 \mathrm{mg} / \mathrm{kg}$ for 6 weeks [9], $30 \mathrm{mg} / \mathrm{kg}$ for 3 weeks [11] and $100 \mathrm{mg} / \mathrm{kg}$ for 8 weeks [14]. 
All these observations indicate that melatonin plays an important role in metabolic processes and may be involved in the pathogenesis of hyperalimentation syndromes. Results of animal studies cannot be directly related to humans, if for no other reason than that of different doses, the duration of melatonin supplementation and divergence of results. For instance, a high-fat diet decreased melatonin secretion in rats [15], while in obese humans significantly higher nocturnal secretion of this hormone was observed [16]. There are also differences in the assessment of population weight status [17].

These differences suggest to conduct further clinical studies on the role of melatonin in the pathogenesis of obesity.

The aim of our study was to evaluate the effect of melatonin supplementation on the weight status of women during the postmenopausal period.

\section{Material and methods}

The study included 56 women, aged 51-65 years (mean $56.9 \pm 5.3$ ), 5-11 years after the last menstrual period and 25 healthy women, aged 27-36 years (mean $31.2 \pm 3.9$ ). The research was performed in 2008-2012 in the autumn-winter period (September-April) when the seasonal rhythm of melatonin secretion is reduced. The physical examination (medical history) included an assessment of their emotional state using the Hamilton Depression Rating Scale (HAM-D). The quality of sleep was estimated by Insomnia Severity Index (ISI) with our own modification, replacing the evaluation of the quality of life (0-4 points) with the assessment of the shortening of sleeping time.

The physical examination included the measurement of the increase in body weight, and waist and hip circumference. These results were used to calculate the body mass index (BMI) and the waist/hip ratio (WHR).

On the basis of the above mentioned tests, the patients were divided into three groups:

- group I (control) - 25 women with normal body weight, without menstrual and sleep disorders,

- group II - 26 postmenopausal women with normal body weight (BMI less than $24.9 \mathrm{~kg} / \mathrm{m}^{2}$ ),

- group III - 30 postmenopausal women with high body weight (BMI of $25.2-34.9 \mathrm{~kg} / \mathrm{m}^{2}$, mean $31.2 \mathrm{~kg} / \mathrm{m}^{2}$ ).

Women enrolled in group II and group III, in whom the weight gain occurred after menopause, and who did not use hormone replacement therapy (HRT) and felt discomfort in the form of sleep disorders and increased appetite were included into the investigations on the effect of melatonin on sleep quality and nutritional status.

Initially routine laboratory tests were performed: blood cell count, serum levels of urea, creatinine, glucose, glycosylated hemoglobin, cholesterol, triglycerides, amylase, lipase, bilirubin, alanine and aspartate aminotransferase, TSH, 17- $\beta$-estradiol and prolactin by immunoassay (Ortho-Clinical Diagnostics - Johnson \& Johnson Co. kit) and FSH (Vitros Product antibodies). The concentration of sulphate 6-hydroxymelatonin (6-HMS) was determined in daytime (7:00-22:00) and at night (22:00-7:00) in urine fractions by immunoenzymatic method using IBL kit (No: RE 59131). On the day of the testing, women remained on a liquid diet ( $\mathrm{Nu}$ tridrinks, Nutricia) $3 \times 400 \mathrm{ml}$ of $1800 \mathrm{kcal}$, and $1500 \mathrm{ml}$ of isotonic water. Moreover, from 22:00 to 7:00 they remained in bed and could use dim or red light for no longer than 10 minutes. Urine fractions were frozen after centrifugation at $-70^{\circ} \mathrm{C}$ until 6 -HMS determination (not longer than 6 months).

Exclusion criteria include moderate depression (more than 9 points on the HAM-D), diabetes, hypertension, liver, kidney, thyroid disorders and other diseases requiring pharmacotherapy.

Women qualified for the study gave their written consent. The approval of the Bioethics Committee of the Medical University of Lodz was also obtained. The study was conducted in accordance with the principles of the Declaration of Helsinki.

After obtaining the material for tests, the women were recommended a standardized and balanced diet (1500 kcal) and melatonin administration at a dose of $5 \mathrm{mg}$ at 21:00 as well as follow-up examinations after $4,8,12,16,20$ and 24 weeks.

After 24 weeks an extended follow-up examination was performed including the assessment of the quality of sleep, BMI and WHR.

In the statistical analysis, Student's $t$-test was used to compare the means for normal distribution or Kruskal-Wallis and Mann-Whitney nonparametric tests to compare the results of two different samples.

The linear correlation coefficient $r$ was used to determine the relationship between the variables. The calculations were made with STATISTICA 9.1 Stat Soft Polska.

\section{Results}

The study included patients after exclusion of any organic and metabolic diseases. Patients in group II and III showed similar mild levels of anxiety and symptoms of depression and a significant degree of sleep disorders (Table I).

They did not differ in the assessment of the results of laboratory tests, particularly in the range of the concentration of FSH, $17 \beta$-estradiol and markers of carbohydrate and lipid metabolism.

Differences were related to body mass index, which in group II was $22.07 \pm 1.48$ and in group III $-29.62 \pm$ $3.69(p<0.001)$ (Table II).

Furthermore, in the group of obese women, lower urinary 6-HMS excretion was observed at night com- 
Tab. I. General characteristics of women enrolled in the study and preliminary study results

\begin{tabular}{|c|c|c|c|}
\hline Characteristics & Group I $(n=25)$ & Group II $(n=26)$ & Group III $(n=30)$ \\
\hline Age (years) & $31.2 \pm 3.9$ & $55.6 \pm 4.9$ & $58.2 \pm 5.6$ \\
\hline BMI $\left(\mathrm{kg} / \mathrm{m}^{2}\right)$ & $21.94 \pm 1.16$ & $22.07 \pm 1.48$ & $29.62 \pm 3.69^{\star \star *}$ \\
\hline $\mathrm{FSH}(\mathrm{mlU} / \mathrm{ml})$ & $13.24 \pm 5.06$ & $79.8 \pm 21.35^{\star \star * *}$ & $91.23 \pm 19.82^{\star \star \star *}$ \\
\hline $17 \beta$-estradiol (pg/ml) & $49.6 \pm 19.26$ & $15.30 \pm 4.91^{* \star *}$ & $12.48 \pm 5.16^{\star \star *}$ \\
\hline 6-HMS - D & $8.58 \pm 2.86$ & $7.73 \pm 2.04$ & $7.18 \pm 2.46$ \\
\hline 6-HMS - N & $27.45 \pm 7.94$ & $25.22 \pm 7.34$ & $12.41 \pm 5.00^{* * *}$ \\
\hline GFR (ml/min) & $103.6 \pm 10.1$ & $99.0 \pm 6.7$ & $96.8 \pm 11.4$ \\
\hline
\end{tabular}

${ }^{* \star} p<0.01,{ }^{* * *} p<0.001$

$\mathrm{BMI}$ - body mass index, FSH - follicle-stimulating hormone, 6-HMS - 6-hydroxymelatonin sulphate, $\mathrm{D}$ - excretion during the day: $\mu \mathrm{g} / 15 \mathrm{~h}, \mathrm{~N}-\mathrm{nocturnal}$ excretion: $\mu \mathrm{g} / 9 \mathrm{~h}$

Tab. II. Tested characteristics of women enrolled in the study

\begin{tabular}{lccccc}
\hline Tested characteristics & \multicolumn{2}{c}{ Group II } & & Group III \\
\cline { 2 - 6 } & A & B & A & B \\
\hline ISI (points) & $13.27 \pm 1.93$ & $10.35 \pm 1.50^{* \star *}$ & $14.53 \pm 2.56$ & $9.80 \pm 1.71^{* \star *}$ \\
\hline BMI (points) & $22.07 \pm 1.48$ & $21.86 \pm 1.28^{*}$ & $29.62 \pm 3.69$ & $27.88 \pm 3.14^{* \star}$ \\
\hline WHR (cm) & $0.75 \pm 0.036$ & $0.74 \pm 0.035$ & $0.88 \pm 0.036$ & $0.87 \pm 0.035$ \\
\hline
\end{tabular}

${ }^{\star} p<0.05,{ }^{* * *} p<0.001$

ISI - Insomnia Severity Index, BMI - body mass index, WHR - waist-hip ratio

Tab. III. Relation between the tested characteristics

\begin{tabular}{llcccc}
\hline Relation between & \multicolumn{2}{c}{ Group II } & \multicolumn{2}{c}{ Group III } \\
\cline { 3 - 6 } & & $\begin{array}{c}\text { Correlation } \\
\text { coefficient }(r)\end{array}$ & $\begin{array}{c}\text { Statistical } \\
\text { significance }\end{array}$ & $\begin{array}{c}\text { Correlation } \\
\text { coefficient }(r)\end{array}$ & $\begin{array}{c}\text { Statistical } \\
\text { significance }\end{array}$ \\
\hline 6-HMS - D & ISI & -0.594 & $<0.001$ & -0.707 & $<0.001$ \\
\cline { 2 - 6 } & BMI & -0.020 & $>0.05$ & -0.651 & $<0.001$ \\
\cline { 2 - 6 } & WHR & 0.022 & $>0.05$ & -0.322 & $>0.05$ \\
\hline 6-HMS - N & ISI & -0.810 & $<0.001$ & -0.821 & $<0.001$ \\
\cline { 2 - 6 } & BMI & -0.105 & $>0.05$ & -0.756 & $<0.001$ \\
\cline { 2 - 6 } & WHR & 0.032 & $>0.05$ & -0.339 & $>0.05$ \\
\hline
\end{tabular}

6-HMS - 6-hydroxymelatonin sulphate, D - excretion during the day: $\mu \mathrm{g} / 15 \mathrm{~h}, \mathrm{~N}$ - nocturnal excretion: $\mu \mathrm{g} / 9 \mathrm{~h}, \mathrm{ISI}$ - Insomnia Severity Index, BMI - body mass index, WHR - waist-hip ratio

pared to patients without obesity $-25.22 \pm 7.34 \mu \mathrm{g} / \mathrm{h}$ and $12.41 \pm 5.00 \mu \mathrm{g} / \mathrm{h}(p<0.001)$, respectively; no such differences were found during the day.

In both investigated groups (II, III) of postmenopausal women, a negative correlation was detected between urinary 6-HMS excretion and the degree of sleep disorders (Table III).

In a detailed analysis, sedative effect of melatonin was found to be assessed by the patients in different ways. The majority (64.2\%) reported that melatonin improved the quality of sleep from the beginning of its administration. The remaining patients (35.8\%) did not feel any significant improvement in the first month of supplementation. This group comprised women who previously took other hypnotic drugs. These disorders, particularly in the form of breaks and shortening of the duration of sleep significantly improved after 6-8 weeks of melatonin administration.

A statistically significant improvement was observed in all patients after 24 weeks; the number of points decreased in group II from $13.27 \pm 1.93$ to 10.35 \pm 1.50 ( $p<0.001)$, and in group III from $14.53 \pm 2.56$ to $9.80 \pm 1.71$ ( $p<0.001)$ (Fig. 1).

The initial examination revealed a negative correlation between urinary 6-HMS excretion and body mass index (Table II). Melatonin supplementation contributed to the reduction of body weight in obese women and after 16 weeks, BMI decreased from $29.62 \pm 3.69$ to $27.88 \pm 3.14(p<0.001)$. The tendency to the reduction in body weight was also observed in group II of 


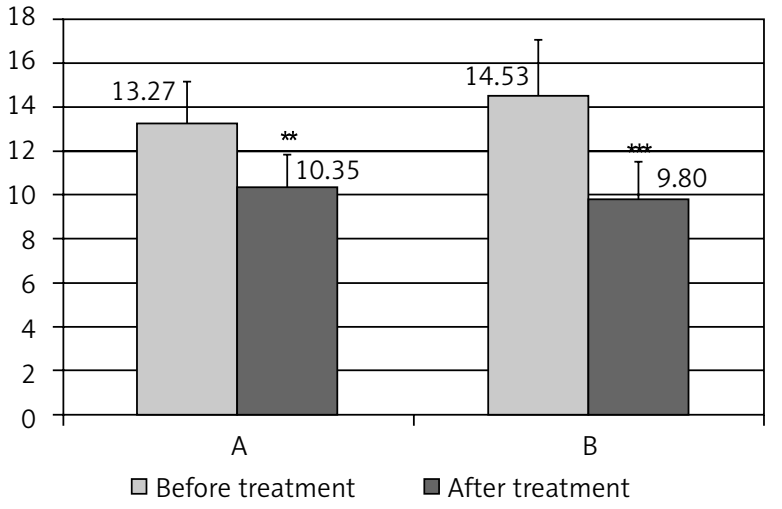

Fig. 1. Insomnia severity index (ISI) before and after melatonin therapy in the groups of postmenopausal women of normal body weight (A) and with obesity (B); ${ }^{* *} p<0.01,{ }^{* * *} p<0.001$

women without obesity (Fig. 2). Waist-hip ratio values exhibited similar tendency but the results did not differ statistically (Fig. 3).

Melatonin was well tolerated, only 4 women out of $56(7.1 \%)$ reported general fatigue in the morning in the first two weeks of the treatment but without the need of discontinuation of the therapy or dose reduction.

\section{Discussion}

The results of many experimental studies and clinical trials suggest that in the case of obesity, the circadian and seasonal rhythm of melatonin secretion is disturbed [18, 19]. Lower levels of melatonin secretion in the autumn-winter period can increase appetite and lead to weight gain [20]. Weight gain and the increase in the level of high-density lipoproteins was found in humans exposed to white light at night $[18,21]$.

Many researchers suggest the use of melatonin in the treatment of obesity [22-24], but still there are no extended research studies supporting this view. Optimization of melatonin doses, which should be provided to patients with varying degrees of obesity, still remains an open and controversial issue. The doses applied in animal experimental studies are often too high and their effects on other functions of the human body are not predictable.

Melatonin supplementation is justified in patients with its deficiency. The secretion of melatonin decreases with age and this process begins as early as after 30 years of age.

It was observed that the reduction in melatonin secretion mainly concerns postmenopausal women; this convergence of biological processes resulted in the introduction of the term "melatoninpause" $[25,26]$.

Toffol et al. [27] confirmed the decreased secretion of melatonin in postmenopausal women but they found no correlation between its level and the level of FSH and estradiol in the serum and also the BMI values.

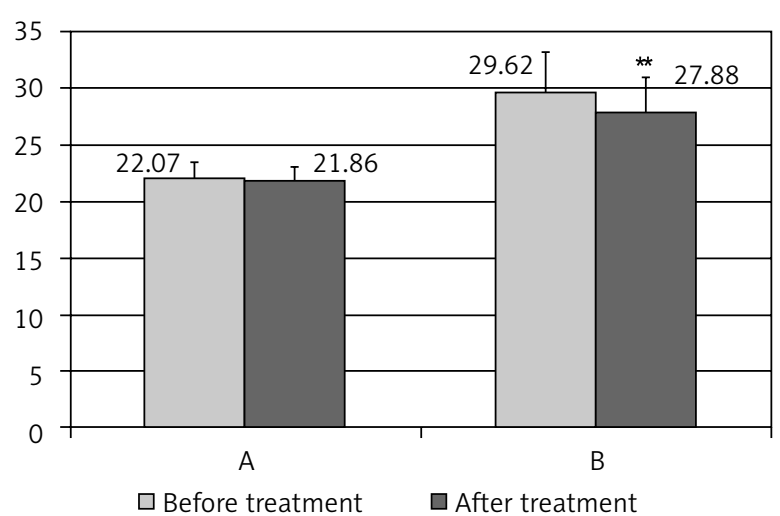

Fig. 2. Body mass index (BMI) before and after melatonin therapy in the groups of postmenopausal women of normal body weight $(\mathrm{A})$ and with obesity $(\mathrm{B}) ;{ }^{* *} p<0.01$

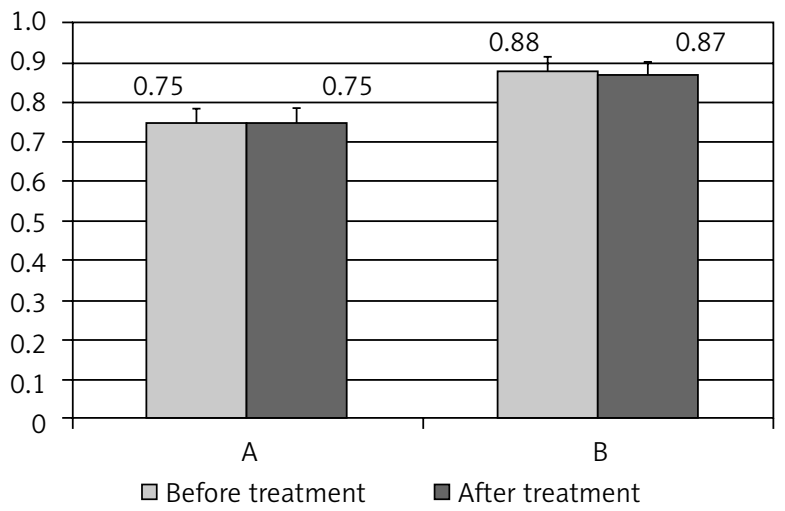

Fig. 3. Waist/hip ratio (WHR) before and after melatonin therapy in the groups of postmenopausal women of normal body weight (A) and with obesity (B)

Blaicher et al. [28] reported decreased urinary 6-HMS excretion in obese postmenopausal females.

The results of our studies indicate that a decrease in the activity of the melatoninergic system particularly at night may be one of the reasons for the tendency to gain weight in postmenopausal women.

The mechanism of the beneficial effect of melatonin on the nutritional status is not clear, but the negative correlation between BMI and urinary 6-HMS excretion, both during the day and at night, is distinct.

In most women the body weight decreased and there was no tendency for its gain. A balanced diet under the supervision of doctors could not be without significance.

Decreased appetite and the improvement of the quality of sleep could have a significant impact on the nutritional status. Estrogen deficiency is considered to be the cause of sleep disorders in postmenopausal women but reduced secretion of melatonin at night may play an important role in this regard. Prolongation of sleep could result in an increase of melatonin secretion at night and melatonin supplementation strengthened the physiological action of this hormone. 
The applied melatonin doses seem to be sufficient for the regulation of complex metabolic disorders associated with menopause. The effect of increased doses of MEL and longer supplementation require further studies on larger groups of patients.

To conclude, it may be stated that the results of our studies and of the studies of many other researchers indicate that melatonin can be safely and permanently used in combined treatment of obesity in postmenopausal women.

\section{Conclusions}

1. Postmenopausal women demonstrate reduced nocturnal secretion of melatonin.

2. Melatonin supplementation has a beneficial effect on the quality of sleep and reduction of body weight.

\section{Acknowledgement}

The study was financed by the grant of MNiSW NN 4025437/40.

\section{Disclosure}

\section{Authors report no conflict of interest.}

\section{References}

1. Reiter RJ, Tan DX, Feuntes-Broto L. Melatonin: a multitasking molecule. Prog Brain Res 2010; 181: 127-151.

2. Brambilla F, Fraschini F, Esposti G, et al. Melatonin circadian rhythm in anorexia nervosa and obesity. Psychiatry Res 1988; 23: 267-276.

3. Ferrari E, Foppa S, Bossolo PA, et al. Melatonin and pituitary - gonadal function in disorders of eating behavior. J Pineal Res 1989; 7: 115-124.

4. Peschke E. Melatonin, endocrine pancreas and diabetes. J Pineal Res 2008; 44: 26-40.

5. Picinato MC, Haber EP, Carpinelli AR, et al. Daily rhythm of glucose-induced insulin secretion by isolated islets from intact and pinealectomized rat. J Pineal Res 2002; 33: 172-177.

6. Rasmussen DD, Boldt BM, Wilkinson CW, et al. Daily melatonin administration at middle age suppresses male rat visceral fat, plasma leptin and plasma insulin to youthful levels. Endocrinology 1999; 140: 1009-1012.

7. Rios-Lugo MJ, Cano P, Jimenez-Ortega V, et al. Melatonin effect on plasma adiponectin, leptin, insulin, glucose, triglycerides and cholesterol in normal and high fat-fed rats. J Pineal Res 2010; 49: 342-348.

8. Agil A, Navarro-Alarcón M, Ruiz R, et al. Beneficial effects of melatonin on obesity and lipid profile in young Zucker diabetic fatty rats. J Pineal Res 2011; 50: 207-212.

9. Favero G, Lonati C, Giugno L, et al. Obesity-related dysfunction of the aorta and prevention by melatonin treatment in ob/ob mice. Acta Histochem 2013; 115: 783-788.

10. Wolden-Hanson T, Mitton DR, McCants RL, et al. Daily melatonin administration to middle-aged male rats suppresses body weight, intraab- dominal adiposity, and plasma leptin and insulin independent of food intake and total body fat. Endocrinology 2000; 141: 487-497.

11. Prunet-Marcassus B, Desbazeille $M$, Bros A, et al. Melatonin reduces body weight gain in Sprague Dawley rats with diet-induced obesity. Endocrinology 2003; 144: 5347-5352.

12. Nduhirabandi F, Du Toit EF, Blackhurst D, et al. Chronic melatonin consumption prevents obesity-related metabolic abnormalities and protects the heart against myocardial ischemia and reperfusion injury in a prediabetic model of diet-induced obesity. J Pineal Res 2011; 50: 171-182.

13. Zanuto R, Siqueira-Filho MA, Caperuto LC, et al. Melatonin improves insulin sensitivity independently of weight loss in old obese rats. J Pineal Res 2013; 55: 156-165.

14. Sartori C, Dessen P, Mathieu C, et al. Melatonin improves glucose homeostasis and endothelial vascular function in high-fat diet-fed insulinresistant mice. Endocrinology 2009; 150: 5311-5317.

15. Cano P, Jimenez-Ortega $V$, Larrad $A$, et al. Effect of a high-fat diet on 24-h pattern of circulating levels of prolactin, luteinizing hormone, testosterone, corticosterone, thyroid-stimulating hormone and glucose and pineal melatonin content in rats. Endocrine 2008; 33: 118-125.

16. Mantele S, Otway DT, Middleton B, et al. Daily rhythms of plasma melatonin, but not plasma leptin or leptin mRNA, vary between lean, obese and type 2 diabetic men. PLoS One 2012; 7: 1-8.

17. Shabnam AA, Homa K, Reza MT, et al. Cut-off points of waist circumference and body mass index for detecting diabetes, hypercholesterolemia and hypertension according to National Non-Communicable Disease Risk Factors Surveillance in Iran. Arch Med Sci 2012; 8: 614-621.

18. Bartness TJ, Demas GE, Song CK. Seasonal changes in adiposity: the roles of the photoperiod, melatonin and other hormones and sympathetic nervous system. Exp Biol Med 2002; 227: 363-376.

19. Reiter RJ, Tan DX, Korkmaz A, et al. Obesity and metabolic syndrome: association with chronodisruption, sleep depivation and melatonin suppression. Ann Med 2012; 44: 564-577.

20. Sato M, Kanikowska D, Iwase $S$, et al. Seasonal differences in melatonin concentrations and heart rates during sleep in obese subjects in Japan. Int J Biometeorol 2013; 57: 743-748.

21. Obayashi K, Saeki K, Iwamoto J, et al. Exposure to light at night, nocturnal urinary melatonin excretion and obesity/dyslipidemia in the elderly: a cross - sectional analysis of the HEILO-KYO study. J Clin Endocrinol Metab 2013; 98: 337-344.

22. Cardinali DP, Cano P, Jimenez-Ortega V, et al. Melatonin and the metabolic syndrome: physiopathologic and therapeutical implications. Neuroendocrinology 2011; 93: 133-142.

23. Rastmanesh R, de Bruin PF. Potential of melatonin for the treatment or prevention of obesity: an urgent need to include weight reduction as a secondary outcome in clinical trials of melatonin in obese patients with sleep disorders. Contemp Clin Trials 2012; 33: 574-575.

24. Srinivasan V, Ohta Y, Espino J, et al. Metabolic syndrome, its pathophysiology and the role of melatonin. Recent Pat Endocr Metab Immune Drug Discov 2013; 7: 11-25.

25. Vakkuri O, Kivela A, Leppaluoto J, et al. Decrease in melatonin precedes follicle-stimulating hormone increase during perimenopause. Eur J Endocrinol 1996; 135: 188-192.

26. Okatani Y, Morioa N, Wakatsuki A. Changes in nocturnal melatonin secretion in perimenopausal women: correlation with endogenous estrogen concentrations. J Pineal Res 2000; 28: 111-118.

27. Toffol E, Kalleinen N, Haukka J, et al. Melatonin in perimenopausal and postmenopausal women: associations with mood, sleep, climacteric symptoms and quality of life. Menopause 2014; 21: 493-500.

28. Blaicher W, Speck E, Imhof MH, et al. Melatonin in postmenopausal females. Arch Gynecol Obstet 2000; 263: 116-118. 